\title{
Simple methods for immobilizing titania into pumice for photodegradation of phenol waste
}

\author{
Slamet $^{1} \cdot$ Setiadi $^{1} \cdot$ Dewi Tristantini $^{1} \cdot$ Eny Kusrini $^{1} \cdot$ Davin Philo $^{1}$
}

Received: 26 September 2016 / Accepted: 23 April 2018 / Published online: 21 May 2018

(c) The Author(s) 2018

\begin{abstract}
Simple methods for immobilizing titania into pumice were applied, where the products had been used for the application of photodegrading phenol waste. There were two simple methods, which were used separately: solvent evaporation and dip coating. Based on titania sol stability test, it was observed that acid condition would be favourable for immobilizing titania unto pumice, and preferable dispersion medium for solvent evaporation method was ethanol, while dip coating method was better to use aquadest (water). Prepared samples were characterized by FE-SEM, EDX, XRD, and BET analysis. Mechanical strength and photodegradation tests were conducted to observe the quality and ability of immobilized photocatalyst. Experimental results show that both methods had produced mechanically strong immobilized titania, where dip coating method tended to produce homogen solid film of titania on the outer surface of pumice, while solvent evaporation method could deliver titania deeper unto the inner part of the pumice. Catalyst detachment level of immobilized titania nanotube by dip coating method was $4.6 \%$, while the one of the products of solvent evaporation method was $5.0 \%$. Furthermore, in terms of photocatalytic activity, product of dip coating method gave slightly better performance (with $28 \%$ of phenol elimination after $240 \mathrm{~min}$ ) than the product of solvent evaporation method (24\%), due to shading effect, mostly occurring to immobilized catalyst in the inner part of the pumice.
\end{abstract}

Keywords Immobilization $\cdot$ Phenol $\cdot$ Photodegradation $\cdot$ Pumice $\cdot$ Simple methods $\cdot$ Titania

\section{Introduction}

These days, the governments of several countries have put great attention on the industrial waste, thus issuing some strict regulations about waste specification prior to discharge to the environment. This action is taken because most of the industrial wastes usually contain hazardous components, such as phenol and its derivatives which are very toxic, carcinogenic, and resistant to degradation due to their high solubility and stability in water $[1,2]$. Various methods for removal of phenol components have been widely studied and developed, including adsorption, biodegradation, extraction, and oxidation, as in ozonation and photocatalysis [3].

Among all of the methods mentioned above, advanced oxidation process (AOP) using semiconductor

Slamet

slamet@che.ui.ac.id

1 Department of Chemical Engineering, Faculty of Engineering, Universitas Indonesia, Depok 16424, Indonesia nano-photocatalyst, such as titania $\left(\mathrm{TiO}_{2}\right)$, appears as one promising technology [4-7]. The main advantage in using AOP is that it can transform the toxic organic pollutants into non-toxic or less toxic products like $\mathrm{CO}_{2}$ [3]. Nowadays, photocatalyst titania has been widely studied and developed for the elimination of organic pollutants, e.g., dyes [5, 8], aniline [9], toluene [10] and phenol [1, 11-13]. However, the utilization of nanosized photocatalyst encounters a major problem, i.e., the difficulty of separating the catalyst after use $[13,14]$. Many techniques have been applied to solve this problem by immobilizing titania unto various supports, such as silica [10,15], polymer [16], activated carbon [11], stainless steel [17], zeolite [4], and pumice [18].

Especially for field application, the usage of floating material like pumice as the support for catalyst is very promising, due to the potency of utilizing solar energy effectively [19]. Moreover, the abundance of pumice (especially in Indonesia) and its relatively low price make pumice really applicable for industrial purpose. However, a study to investigate and obtain immobilized photocatalyst with good mechanical strength, especially on porous support such as 
pumice, is still infrequent. In this regard, many studies have found it difficult to maintain the catalyst on certain support, thus causing significant decrease of photocatalytic efficiency due to the loss of catalyst, especially for long-term usage $[10,18]$.

To produce mechanically strong immobilized photocatalyst, a proper method is indispensable. Several sophisticated methods for immobilizing titania unto certain substrates have been studied and developed, e.g., chemical vapor deposition [20], electrophoretic deposition [21], sputtering [15], and pulsed laser deposition [22]. However, considering the complexity and high cost of those methods, it is more desirable to develop any other simpler method to improve the efficiency of the process. Of the various immobilization methods existing, there are two comparatively simple methods: solvent evaporation [23] and dip coating [17].

As mentioned before, it is essential to optimize the immobilization process to produce immobilized titania which adheres strongly on the support. One of the methods to manipulate the attachment process of titania unto certain support is by controlling the dispersion of titania nanoparticles to obtain stable sol [24]. Furthermore, there are several ways which have also been developed to utilize the photocatalyst sol effectively: reducing the size of the catalyst particles [25] and forming electrostatic bonds based on surface charge differences [12]. Interestingly, it is possible to perform both ways simultaneously for titania and pumice case, by adjusting the acidity of photocatalyst sol. Such strategy has been successfully performed and will be discussed on this report.

This study was aimed to optimize and compare two simple immobilization methods, i.e., solvent evaporation and dip coating In the first place, the effects of dispersion medium, $\mathrm{pH}$, and temperature to the stability level of titania sol were investigated to determine proper condition for each method. Thereafter, mechanical strength and phenol photodegradation performance of the immobilized titania were also observed and analyzed.

\section{Experimental}

\section{Materials}

$\mathrm{TiO}_{2} \mathrm{P} 25$ with a crystallographic mode of $79 \%$ anatase and $21 \%$ rutile, a $53.6 \mathrm{~m}^{2} \mathrm{~g}^{-1}$ BET surface area [26] and an average particle size of $21 \mathrm{~nm}$, used as raw materials, was bought from Evonic Industries. Sodium hydroxide pellet and fuming hydrochloric acid for preparation of titania nanotube (TiNT) were purchased from Merck and Mallinckrodt, respectively. Aquadest and ethanol, used as dispersion medium for photocatalyst sol, were bought from PT. Brataco. Floatable pumices used as the support were natural pumices obtained from
Lombok, Indonesia. Phenol solid to synthesize the pollutants sample were purchased from Merck.

\section{Photocatalyst titania nanotube (TiNT) preparation}

There were two types of titania used in this study: titania nanotube (TiNT) and titania nanoparticle P25. The preparation of TiNT was similar to the previous study [27]. Firstly, $6 \mathrm{~g}$ of titania $\mathrm{P} 25$ powder was dissolved into $300 \mathrm{~mL}$ of $10 \mathrm{M}$ $\mathrm{NaOH}$ and ultrasonicated for $30 \mathrm{~min}$. Thereafter, the mixture underwent hydrothermal treatment at $130{ }^{\circ} \mathrm{C}$ for $6 \mathrm{~h}$ and was stirred at $600 \mathrm{rpm}$ using a Teflon-lined autoclave equipped with adjustable rotating speed mixer. The sample obtained was washed with acid $(0.2 \mathrm{~N} \mathrm{HCl})$ repeatedly until it reached $\mathrm{pH} 2$ and then with aquadest until approximately $\mathrm{pH} 5$. TiNT powder was then obtained after drying at $80^{\circ} \mathrm{C}$ for $6 \mathrm{~h}$ and calcination at $500{ }^{\circ} \mathrm{C}$ for $1 \mathrm{~h}$.

\section{Stability test of photocatalyst sol}

Stability test of photocatalyst sol was performed to measure the stability level of the photocatalyst sol which would be used to coat pumices. Firstly, the photocatalyst $\left(\mathrm{TiO}_{2} \mathrm{P} 25\right)$ sols with different conditions ( $\mathrm{pH}$, dispersion medium, and temperature) which would be used for immobilizing titania unto pumices were prepared beforehand. Firstly, $0.5 \mathrm{~g}$ of titania was added into $100 \mathrm{~mL}$ of dispersion medium (ethanol or aquadest). The mixture was placed in a Pyrex cylindrical glass with size of $8.5 \mathrm{~cm} \times 5.0 \mathrm{~cm} \times 4.6 \mathrm{~cm}$ (height $\times$ outer diameter $\times$ inner diameter) and total volume of $140 \mathrm{~mL}$. After that, several drops of concentrated $\mathrm{HNO}_{3}$ solution were added to the sol for adjusting the sol acidity until $\mathrm{pH} 2-3$, continued by addition of $0.23 \mathrm{~mL}$ of tetraethyl orthosilicate (TEOS) which worked as adhesive. After stirring for $30 \mathrm{~min}$, ultrasonic treatment for several minutes was then given to the mixture and followed by another stirring for $30 \mathrm{~min}$.

The prepared photocatalyst sol was then left for $3 \mathrm{~h}$ without stirring. From time to time, $3 \mathrm{~mL}$ of the supernatant (the liquid lies on the top of the sol) were drawn. The samples were analyzed by a spectrophotometer UV/Vis Spectroquant Pharo $300(\lambda=420 \mathrm{~nm})$ after diluted ten times. The results will be presented as the ratio of the sample absorbances $(A)$ to absorbance of the sample at initial condition $\left(A_{0}\right)$, which relates to the stability level of the sol.

\section{Synthesis of immobilized titania on pumice}

In this study, synthesis of immobilized titania on pumice was performed by two different methods: solvent evaporation and dip coating. The photocatalyst sol for coating the pumice with titania was prepared beforehand according to the procedures which have been explained above. For solvent 
evaporation method, $19.5 \mathrm{~g}$ of pumices was then added to the sol. The mixture was stirred sufficiently and ultrasonic treated for $5 \mathrm{~min}$ to facilitate the interaction between titania and pumices. Thereafter, heat treatment on $80^{\circ} \mathrm{C}$ was given to the mixture to evaporate all of the dispersion medium.

For dip coating method, $19.5 \mathrm{~g}$ of pre-treated pumices, which had firstly placed on a wire basket was dipped to the sol for 5-10 min. Then the basket was lifted up in certain speed and dried using warm and dry air until the pumices were apparently dry. Then, the dipping-drying cycles were performed repeatedly until all of the sol had been used up to coat the pumices. The products of both immobilization methods were then calcined at $500^{\circ} \mathrm{C}$ for $1 \mathrm{~h}$.

For simplification, when labeling certain immobilized titania, the immobilization method used to synthesize the catalyst will be written as abbreviation following the name of the catalyst: solvent evaporation method will be abbreviated by - SE, while dip coating method by - DC. For example, immobilized TiNT-SE will refer to immobilized TiNT synthesized by solvent evaporation method.

\section{Catalyst characterization}

Catalyst titania and immobilized titania on pumice were further characterized by several analysis methods. The surface morphology and the material composition of samples were analyzed using a field emission-scanning electron microscope FE-SEM FEI INSPECT F50 equipped with energy dispersive X-ray spectroscopy (EDX) AMETEK which was conducted at $20 \mathrm{kV}$ (15 kV for imaging $\mathrm{TiO}_{2} \mathrm{P} 25$ ). The crystalline phase of the TiNT and $\mathrm{TiO}_{2} \mathrm{P} 25$ were determined using powder X-ray diffraction (XRD) analysis. The XRD patterns were obtained using an X-Ray Diffractometer Shimadzu MAXima_X XRD-7000 with $\mathrm{Cu}$ anode tube $(l=0.154184 \mathrm{~nm})$. XRD tube runs at voltage of $40 \mathrm{kV}$ and current of $30 \mathrm{~mA}$, with scanning rate of $2 \%$ min and sampling pitch of $0.02^{\circ}$ in the range of $2 \theta=10^{\circ}-80^{\circ}$. The instrumental control and data processing were handled by an IBM PC/ AT compatible PC.

For better understanding of photocatalyst surface properties, Brunauer-Emmet-Teller (BET) analysis was accomplished to measure the specific surface area and pore size of catalyst. In this study, the BET characterization was performed by employing $\mathrm{N}_{2}$ physical adsorption principle, using Autosorb-6 manufactured by Quantachrome Corp. BET data were automatically retrieved using ASORB6AG software, which is integrated with Autosorb-6. Immobilized titania were only characterized by FE-SEM/EDX and BET analysis, for it was not important to undergo other analysis because the titania used for synthesizing immobilized titania were the same as the catalyst characterized as explained above.

\section{Mechanical strength test of immobilized titania}

Mechanical strength test was performed to measure the mechanical strength of immobilized titania layer on the pumice surface. The test was performed by putting $5 \mathrm{~g}$ of pumices which had been immobilized by titania into $50 \mathrm{~mL}$ of water in a Pyrex cylindrical glass with size of $6 \mathrm{~cm} \times 4.6 \mathrm{~cm} \times 4.2 \mathrm{~cm}$ (height $\times$ outer diameter $\times$ inner diameter) and total volume of $80 \mathrm{~mL}$. Then, the mixture was given ultrasonic treatment for 5 min using ultrasonic bath. Then $3 \mathrm{~mL}$ liquid sample of the mixture was drawn to analyze its turbidity. The sample was analyzed by a spectrophotometer UV/Vis using Spectroquant Pharo 300 $(\lambda=420 \mathrm{~nm})$, to measure the absorbance of each sample. The absorbance of the sample was compared to the absorbance of the photocatalyst sol. The result of this test would be presented as a percentage of catalyst detachment (i.e. the ratio of the absorbance of sample, $A_{\text {sample }}$, and the absorbance of photocatalyst sol, $A_{\text {photocatalyst sol }}$ ).

$\%$ catalyst detachment $=\frac{A_{\text {sample }}}{A_{\text {photocatalyst sol }}} \times 100 \%$.

\section{Photocatalytic performance test}

Performance test was performed by measuring the phenol photodegradation efficiency of catalyst (18 $\mathrm{g}$ for pumices containing immobilized titania and $0.3 \mathrm{~g}$ for pure titania). Phenol solution (10 ppm) was prepared by dissolving solid phenol in $300 \mathrm{~mL}$ of water. The test was performed in cylindrical Pyrex open reactor with size of $12.5 \mathrm{~cm} \times 9.0 \mathrm{~cm} \times 8.6 \mathrm{~cm}$ (height $\times$ outer diameter $\times$ inner diameter) and total volume of $700 \mathrm{~mL}$ which was placed in a closed test box lined by aluminum foil. Mercury lamp PHILIPS HPL-N $250 \mathrm{~W}\left(165 \mu \mathrm{m} / \mathrm{cm}^{2}\right)$ was used as the power source. The reactor was also equipped by a magnetic stirrer to ensure the reaction could take place uniformly and a blower to control the system temperature.

At first, the mixture was stirred for $30 \mathrm{~min}$ without photon irradiation to allow the system for reaching adsorption-desorption equilibrium. The test was further conducted with light irradiation for $240 \mathrm{~min}$ and liquid samples were drawn from time to time. The liquid samples were analyzed using spectrophotometer UV/Vis: Spectroquant Pharo 300 with measured parameter of phenol concentration. The degree of phenol elimination was calculated using following equation:

$$
\begin{aligned}
\% \text { Elimination } & =\left[\left(C_{0}-C_{t}\right) / C_{0}\right] \times 100 \% \\
& =\left[\left(A_{0}-A_{t}\right) / A_{0}\right] \times 100 \%,
\end{aligned}
$$

where $A_{0}$ and $A_{t}$ are the initial and final absorbance (at time $t$ ) of phenol in the solution, which respectively relate to the 
initial and final concentration $\left(C_{0}\right.$ and $\left.C_{t}\right)$ according to the Beer-Lambert Law [28, 29]. In order to study the kinetics of the phenol elimination process, the pseudo first-order kinetics according to the Langmuir-Hinshelwood model was used [28, 30]:

$\ln \left(C_{t} / C_{0}\right)=-k t$.

The rate constants $(k)$ were calculated using the slopes of the straight lines found on the plot of $\ln \left(C_{t} / C_{0}\right)$ versus irradiation time $(t)$.

\section{Results and discussion}

\section{Characterization of photocatalyst titania}

Figure 1 illustrates the FE-SEM image of P25 (Fig. 1a) and TiNT (Fig. 1b). Those images show that the $\mathrm{TiO}_{2}$ nanoparticles were successfully modified to $\mathrm{TiO}_{2}$ nanotubes with
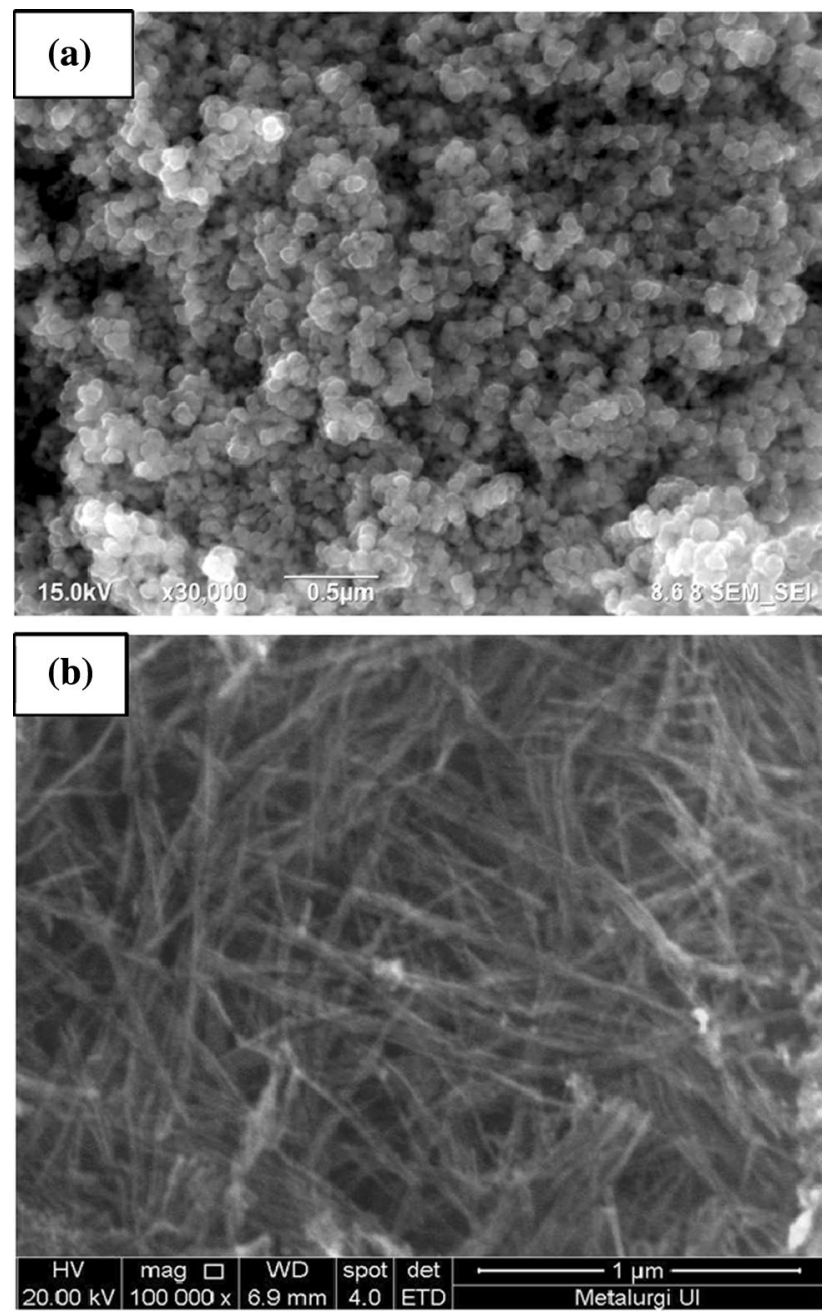

Fig. 1 FE-SEM image of a $\mathrm{TiO}_{2}$ P25 nanoparticles; $\mathbf{b}$ TiNT outer diameter of $24-42 \mathrm{~nm}$. The tubes of TiNT are relatively thin, but long and they are separated perfectly from each other. For some perspective, the prepared TiNT was very potential to be immobilized on the pumice surface; the perfect tube form of TiNT would have relatively wide contact area, enabling it to attach more easily to any surfaces, including pumice surfaces. Intense discussion about the formation of TiNT had been performed on previous study [27].

Figure 2 shows the XRD patterns of $\mathrm{TiO}_{2} \mathrm{P} 25$ and prepared TiNT. The dominant peaks in Fig. 2a at $2 \theta$ of $25.28^{\circ}$, $37.82^{\circ}, 48.08^{\circ}, 53.94^{\circ}$, and $55.06^{\circ}$ are fit to the crystalline structure of anatase $\mathrm{TiO}_{2}$, which correspond to the crystal planes of (101), (004), (200), (105), and (211), respectively (according to JCPDS no. 21-1272). In addition, relatively small peaks at $2 \theta$ of $27.46^{\circ}, 36.08^{\circ}$, and $41.28^{\circ}$ corresponding to (110), (101), and (111) plane of rutile crystalline structure (JCPDS no. 21-1276) was also ascertained for the case of P25. The XRD pattern of TiNT (Fig. 2b) also contains peaks of anatase $\mathrm{TiO}_{2}\left(2 \theta: 25.35^{\circ}, 37.87^{\circ}, 48.10^{\circ}\right.$, $54.03^{\circ}$, and $55.13^{\circ}$ ), but not rutile peak.

For further analysis, crystallite size and anatase fraction of the catalyst were evaluated (see Table 1). The crystallite size of titania phase was estimated from full-width at halfmaximum (FWHM) using the Scherrer equation [28]:

$L=\frac{0.9 \lambda}{\beta \cos \theta}$,

where $L$ is the crystallite size, $\lambda$ is the $\mathrm{X}$-ray wavelength used, $\beta$ is the width of the peak [full width at half maximum (FWHM), expressed in radian], and $\theta$ is the Bragg angel. For

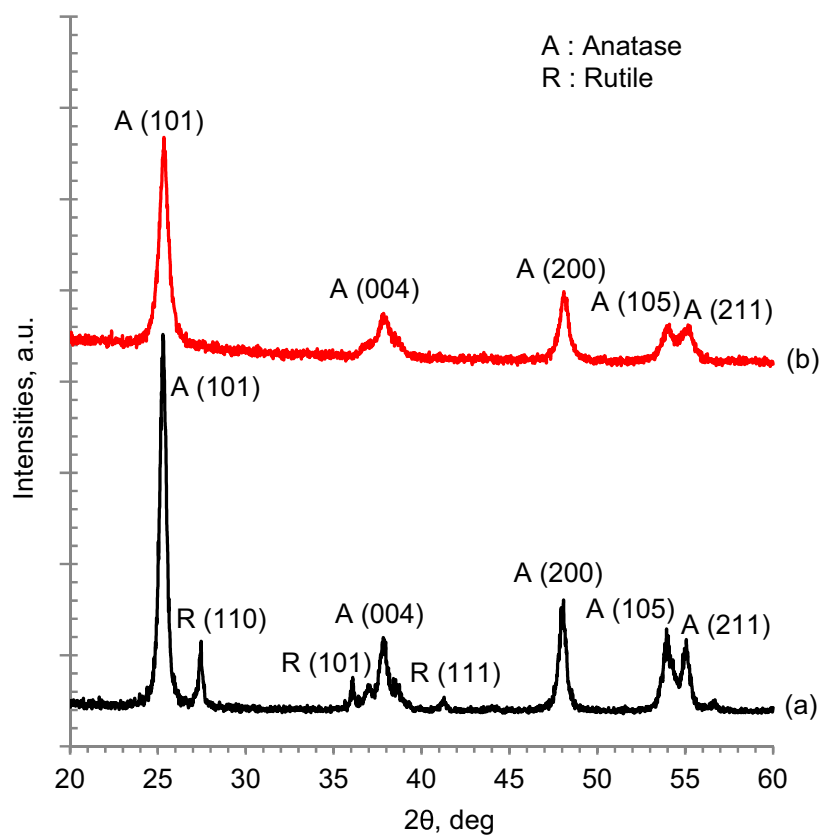

Fig. 2 XRD pattern of photocatalyst: a $\mathrm{TiO}_{2} \mathrm{P} 25$; b TiNT 
Table 1 Crystal size and anatase fraction of photocatalyst

\begin{tabular}{|c|c|c|c|}
\hline \multirow[t]{2}{*}{ Catalyst } & \multicolumn{2}{|c|}{ Crystallite Size $(\mathrm{nm})^{\mathrm{a}}$} & \multirow{2}{*}{$\begin{array}{l}\text { Anatase } \\
\text { fraction } \\
(\mathrm{wt} \%)^{\mathrm{b}}\end{array}$} \\
\hline & Anatase & Rutile & \\
\hline $\mathrm{TiO}_{2} \mathrm{P} 25$ & 20 & 23 & 79 \\
\hline TiNT & 11 & - & 100 \\
\hline
\end{tabular}

${ }^{a}$ Estimated from FWHM of XRD by the Scherrer equation

${ }^{\mathrm{b}}$ Evaluated from the ratio of $I_{\mathrm{R}(110)}$ and $I_{\mathrm{A}(101)}$

the estimation of the crystallite size of anatase phase, the first three peaks $\left(2 \theta: 25.35^{\circ}, 37.87^{\circ}\right.$, and $\left.48.10^{\circ}\right)$ were used and the results were averaged, while for the rutile phase, all three emerging peaks $\left(2 \theta: 27.46^{\circ}, 36.08^{\circ}\right.$, and $\left.41.28^{\circ}\right)$ on the XRD plots were used and the results were also averaged. The fraction of anatase phase was estimated using the following equation [31]:

$X_{\mathrm{A}}=\frac{1}{\left(1+1.265 \frac{I_{\mathrm{R}(110)}}{I_{\mathrm{A}(101)}}\right)}$,

where $X_{\mathrm{A}}$ is the weight fraction of anatase phase, $I_{\mathrm{R}(110)}$ is the peak intensity of rutile (110), and $I_{\mathrm{A}(101)}$ is the peak intensity of anatase (101).

It can be seen that the peaks and crystallite size of TiNT was relatively small (compared to P25), indicating that TiNT was not fully crystallized. It is said that as the peaks and crystallite size of anatase become higher and larger, respectively, the degree of crystallinity also increases [32]. Hydrothermal process in the presence of high concentration $\mathrm{NaOH}$ would convert the $\mathrm{TiO}_{2}$ crystal into amorphous form [33] and turn it into crystal seeds. In this case, the calcination process that took place during the post treatment was believed to influence the phase structures and microstructure of TiNT, by advancing the phase transformation of the amorphous phase unto the anatase phase [34]. However, it seems that the calcination process at $500{ }^{\circ} \mathrm{C}$ was not enough to transform the amorphous titania unto titania with high degree of crystallinity. The rutile phase was not found in TiNT because rutile phase are reported to develop from the anatase phase on higher temperature $\left(800^{\circ} \mathrm{C}\right)[32]$. The absence of rutile phase also verifies that TiNT still contains amorphous phase, since the formation of rutile will take place after all the amorphous phases convert into anatase phase.

BET analysis result for TiNT catalyst is shown in Fig. 3. As can be seen, the shape of TiNT adsorption-desorption curve contains some hysteresis and follows the type IV, which denotes that it has mesoporous structure with pore size of 20-500 $\AA$ [35]. This is in agreement with the measurement result, which indicates average pore size of

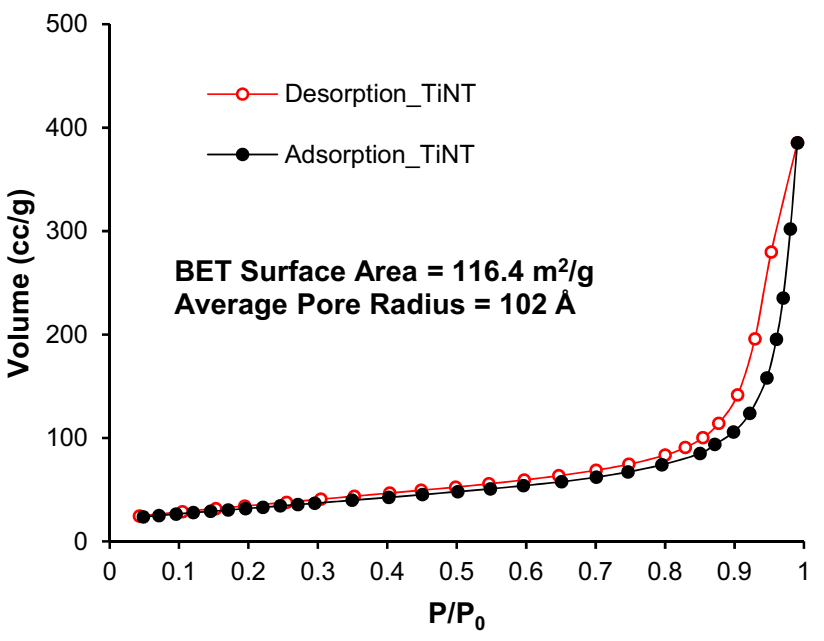

Fig. 3 BET analysis of TiNT

$102 \AA$ A. Moreover, it can be inferred from the hysteresis that the pore shape of TiNT follows A type, that is, cylindrical pore [35].

The surface area of as prepared TiNT was evaluated by employing BET multipoint analysis, resulting the value of $116.4 \mathrm{~m}^{2} / \mathrm{g}$, which indicates a significant improvement compared to its raw material, P25 of which, surface area is $53.6 \mathrm{~m}^{2} / \mathrm{g}$ [26]. The transformation of $\mathrm{TiO}_{2}$ morphology from nanoparticle to nanotube leads to improvement in porosity of the catalyst. Bigger surface area of catalyst might affect positively the photoefficiency of TiNT, especially for photodegradation of organic pollutants.

\section{Stability test of photocatalyst sol}

Mechanical strength of any immobilized titania will be very related to the stability of the sol used. Therefore it is important to review the stability test result beforehand. Figure 4 shows the effects of solution $\mathrm{pH}$ on photocatalyst sol (dispersion medium: aquadest) stability at room temperature $\left(25^{\circ} \mathrm{C}\right) . A / A_{0}$ is the ratio of the absorbance of the sample to the absorbance of the sample at initial condition, which represents the stability level of the sol.

At normal condition, without addition of any acid or base, $\mathrm{pH}$ of the aquadest sol was around 5-6 and it had very low level stability (0.4\%), indicating that almost all of the catalyst particles sank. On the other hand, at acidic condition (pH 2-3), the stability level of the aquadest sol went up to $89 \%$. These occasion might be ascribed to the protonation of titania particles at acidic condition, which would make the surface of titania particles become positively charged. Similar charge between particles of titania would cause the particles to repel each other, thus avoiding agglomeration of the particles [36]. This phenomenon would lead to the 


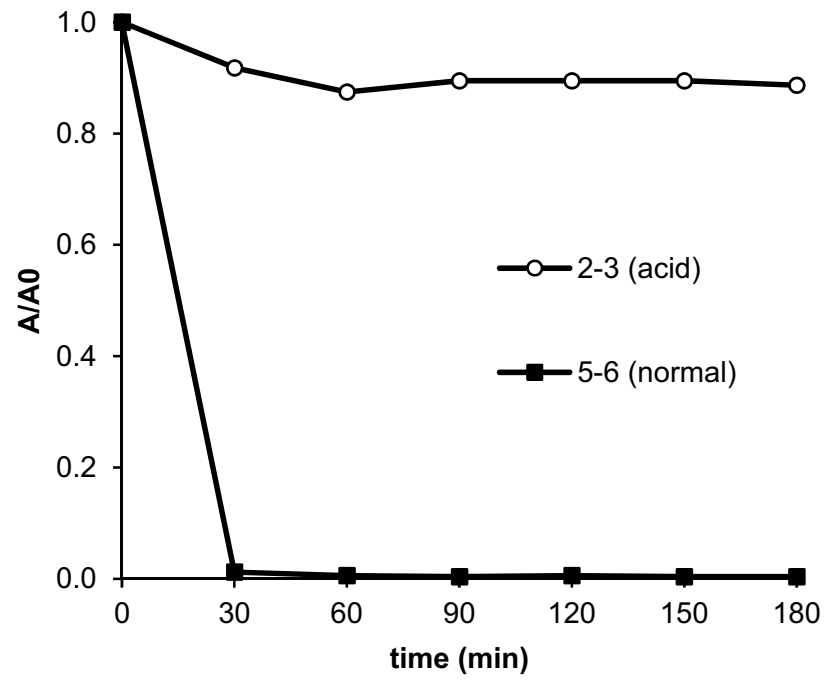

Fig. 4 Effect of $\mathrm{pH}$ on photocatalyst sol stability level (temperature: $25^{\circ} \mathrm{C}$, dispersion medium: aquadest)

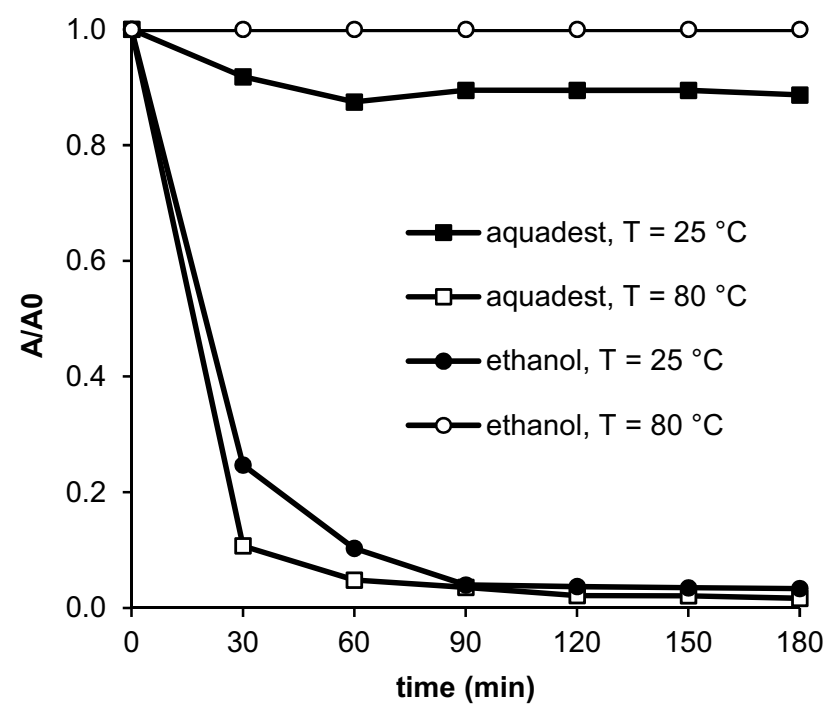

Fig. 5 Effect of temperature and dispersion medium on photocatalyst sol stability level ( $\mathrm{pH} 2-3$ )

breakdown of $\mathrm{TiO}_{2}$ particles into smaller particles and as the result the sol became relatively more stable [25].

It was also necessary to review the stability of sol on different temperature, for the immobilization methods used in this study were conducted at two different temperatures: $25^{\circ} \mathrm{C}$ (room temperature) for dip coating and $80{ }^{\circ} \mathrm{C}$ for solvent evaporation. Figure 5 shows the effect of temperature and dispersion medium on photocatalyst sol stability. It can be seen that at room temperature $\left(25^{\circ} \mathrm{C}\right)$ ethanol sol had relatively low level of stability $\left(A / A_{0}\right.$ is only around $\left.4 \%\right)$. This phenomenon shows that the protonated $\mathrm{TiO}_{2}$ particles were not stable on ethanol media. The possible cause of this phenomenon is that the non-polar molecules of ethanol would repulse the polar (protonated) $\mathrm{TiO}_{2}$ particles, causing them to sink. It is matched with the profile of ethanol sol stability level, where it is not relatively constant until $90 \mathrm{~min}$ of test, but gradually decreases, which indicates the slow sinking process of titania particles (not an immediate process).

At relatively high temperature $\left(80^{\circ} \mathrm{C}\right)$, while aquadest sol experienced a very significant decrease of stability level, where the $A / A_{0}$ becomes $1.68 \%$, stability of ethanol sol on contrary experienced a drastic increase, where $A / A_{0}$ goes up to $100 \%$. Li et al. [37] also find similar result that at relatively higher temperature, ethanol sol has a very stable dispersion rates, while sol which use water as dispersion medium will experience decrease on its stability level.

An increase in the stability level of the ethanol sol occurred as a result of dynamic movement of ethanol molecules which were on boiling phase. It is estimated that the bubbles generated by ethanol could break and stir the $\mathrm{TiO}_{2}$ particles, so the stability of photocatalyst sol could increase dramatically. On the contrary, decrease of aquadest sol stability at high temperatures was caused by the reagglomeration that occurs between $\mathrm{TiO}_{2}$ particles. Based on these results, it can be concluded that for immobilization method which use lower temperature such as dip coating, usage of aquadest as dispersion medium is more favourable. On the other hand, for the immobilization method using higher temperature such as solvent evaporation, ethanol is better to be used as dispersion medium. This issue will be discussed more intensely on the "Mechanical Strength Test" section.

\section{Characterization of immobilized titania on pumice}

In this section, the condition of photocatalyst sol used for the immobilization of titania unto pumice was adjusted to utilize the most stable sol in accordance to the characteristic of each method (based on the result of sol stability test, see Fig. 5). In this case, for solvent evaporation method, the dispersion medium used was ethanol and for dip coating method, aquadest was used, and for both methods acid condition was applied.

Figure $6 \mathrm{a}, \mathrm{b}$ illustrates the FE-SEM images of pumice with two different magnifications $(150 \times$ and $1200 \times$ ), which show that pumice has a porous structure, thus making it as a good support for the catalyst [18, 38]. Furthermore, it can be seen on the FE-SEM images of immobilized TiNTSE (Fig. 6c, d) and immobilized TiNT-DC (Fig. 6e, f), that TiNT catalysts were successfully immobilized on the pumice surface, making the porosity level of the pumice decreased.

Those images also show that the two different methods gave out different results, where solvent evaporation method was seemed to produce immobilized titania which were distributed less evenly compared to dip coating method. In this regard, this occasion might be ascribed to the attachment 
Fig. 6 FE-SEM image of: a pumice $\times 150$; $\mathbf{b}$ pumice $\times 1200$, $\mathbf{c}$ immobilized TiNT-SE $\times 10,000$; $\mathbf{d}$ immobilized TiNTSE $\times 50,000$; e immobilized TiNT-DC $\times 10,000$; f immobilized TiNT-DC $\times 50,000$; g immobilized $\mathrm{TiO}_{2} \mathrm{P} 25-\mathrm{DC}$ $\times 10,000 ; \mathbf{h}$ immobilized $\mathrm{TiO}_{2}$ $\mathrm{P} 25-\mathrm{DC} \times 50,000$
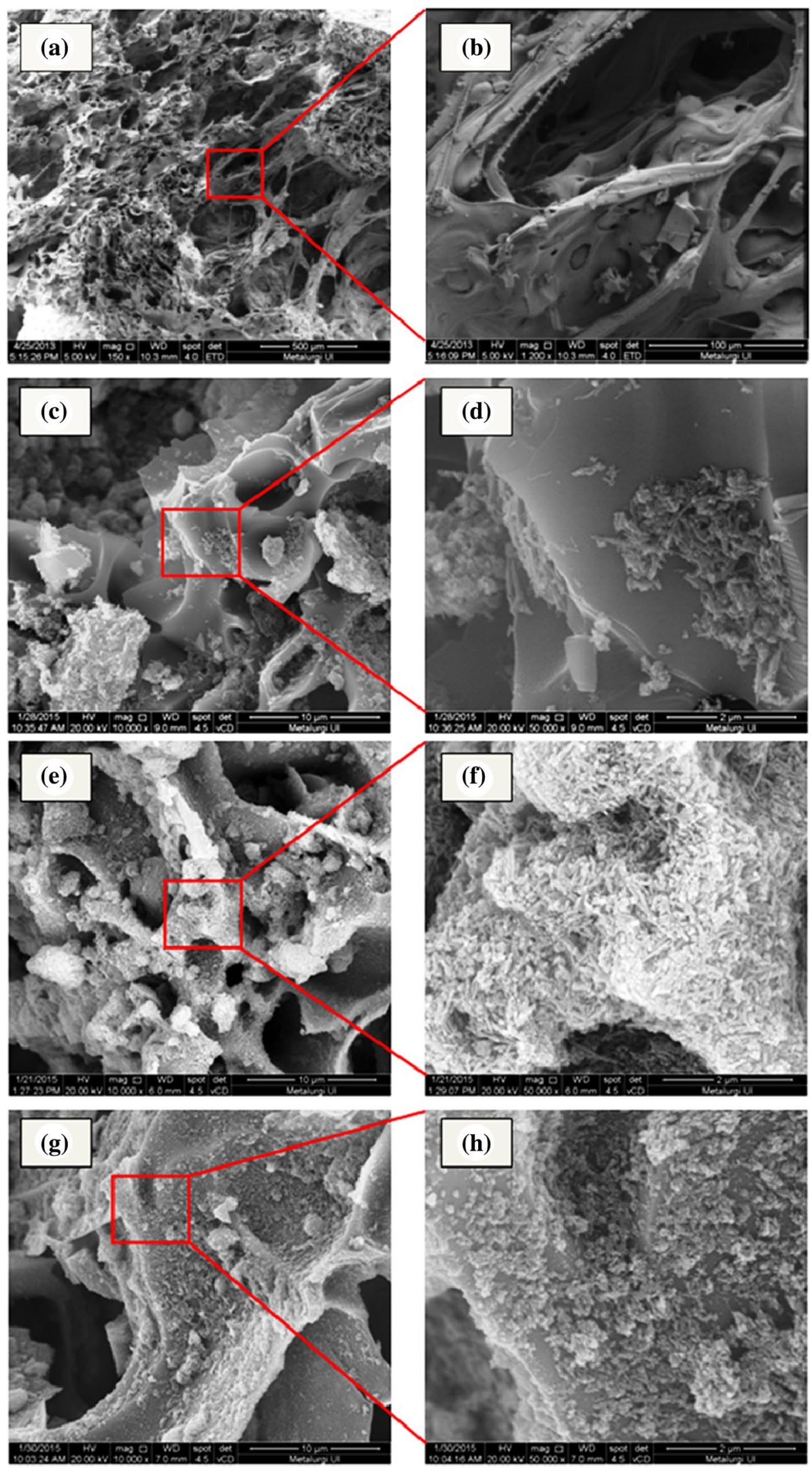
process of titania to pumice surface which was different for each method. For the solvent evaporation method, high temperature was used to evaporate the dispersion medium leaving the titania on the pumice surface. In this case, the boiled solvent moved dynamically and (indirectly) stirred the mixture, thus making the attachment process of titania on pumice was very random. It explains why titania is less spotted on the pumice surface for the case of immobilized TiNT-SE (Fig. 6c, d). However, solvent evaporation method had the advantage to leave all the particles of titania on the pumice, making the loss of titania became inconsiderable. Therefore, it was expected that solvent evaporation method would deliver the titania mostly not on the outer surface of pumice, but deeper into the inner pores of pumice (which would hardly be spotted by FE-SEM analysis). It could happen because of the high mobility of ethanol molecules (on boiling phase) which would give more energy to titania particles and drive them into deeper area of the pumice.

On the contrary, Fig. 6e, f describes how the TiNT catalyst has covered all the outer surface of pumice as the product of dip coating method. It has been reported that dip coating method can produce a thin and controllable layer on the support [36]. Unlike solvent evaporation method, dip coating method was conducted on relatively low temperature. In this condition, the molecules of aquadest would just slightly move in the system, making the interaction between TiNT and pumice occurred mostly on the outer surface of pumice. Therefore, most titania particles were immobilized on the external part of pumice. One benefit to be noticed is dip coating method could produce very homogen layer of catalyst on the surface of support, which would favor the mechanical strength of immobilized titania.

Compared to the FE-SEM image of TiNT (Fig. 1b), the immobilized TiNT (Fig. 6c-f) apparently has shorter and wider tube, with diameter of $40-70 \mathrm{~nm}$. The increase in size (diameter) of TiNT might happen due to the sintering effect $[32,39]$ occured on the second calcination experienced by the immobilized TiNT on pumice. Sintering effect could be described as a phenomenon where the anatase crystallites fused together, forming a relatively larger structure [32]. Before the immobilization step, TiNT had firstly calcined at high temperature to get the crystalline form of titania. On this step, the formation of crystallite phase occurred without accompanied by the sintering process, hence TiNT produced was thin and separated perfectly. Therefore, the tube diameter would also become larger because the tube should adapt to the growth of the anatase crystallite. Qamar et al. [32] get similar phenomenon for TiNT which is calcined at rather high temperature $\left(700{ }^{\circ} \mathrm{C}\right)$.

Figure $6 \mathrm{~g}$, $\mathrm{h}$ shows the FE-SEM images of immobilized $\mathrm{TiO}_{2}$ P25-DC. Similar to the immobilized TiNT-DC, here we also find that $\mathrm{TiO}_{2} \mathrm{P} 25$ particles are distributed uniformly on the pumice surface. However, unlike immobilized
Table 2 EDX analysis on pure pumice and immobilized TiNT

\begin{tabular}{llll}
\hline Element & \multicolumn{2}{l}{ Composition (\% mass) } \\
\cline { 2 - 4 } & Pumice & $\begin{array}{l}\text { Immobilized TiNT- } \\
\text { SE }\end{array}$ & $\begin{array}{l}\text { Immobi- } \\
\text { lized TiNT- } \\
\text { DC }\end{array}$ \\
\hline $\mathrm{O}$ & 43.34 & 51.65 & 43.82 \\
$\mathrm{Na}$ & 4.54 & 4.30 & 3.72 \\
$\mathrm{Al}$ & 9.81 & 8.53 & 8.95 \\
$\mathrm{Si}$ & 34.94 & 30.26 & 33.61 \\
$\mathrm{~K}$ & 3.21 & 2.16 & 2.70 \\
$\mathrm{Ca}$ & 1.23 & - & - \\
$\mathrm{Fe}$ & 2.92 & - & - \\
$\mathrm{Ti}$ & - & 3.11 & 7.21 \\
\hline
\end{tabular}

Table 3 Surface area of the samples by BET analysis

\begin{tabular}{lc}
\hline Samples & $\begin{array}{l}\text { Surface } \\
\text { area } \\
\left(\mathrm{m}^{2} / \mathrm{g}\right)\end{array}$ \\
\hline Pure pumice & 1.039 \\
Immobilized TiNT-SE & 2.095 \\
Immobilized TiNT-DC & 1.612 \\
TiNT & 116.4 \\
\hline
\end{tabular}

TiNT-DC, there are still uncovered area of pumice surface can be found on immobilized P25 cases (Fig. 6e, f). This phenomenon might be attributed to the morphology of each catalyst. Prepared TiNT had long tube morphology, which had relatively greater contact area to be attached to pumice surface compared to titania P25 which had particle morphology, thus enabling TiNT to fill relatively more space on the pumice surface. This result is also consistent with BET result that TiNT had surface area twice of $\mathrm{TiO}_{2} \mathrm{P} 25$.

Table 2 shows the EDX analysis on pure pumice, immobilized TiNT-SE and immobilized TiNT-DC. From the EDX result, it can be seen that the main component of pumice is oxygen $(\mathrm{O})$, silica $(\mathrm{Si})$, and Aluminium $(\mathrm{Al})$. Considerable amount of silica in the form of $\mathrm{SiO}_{2}$ [38] would be the main factor for negative charge of pumice surface. The presence of Ti element in pumice containing TiNT shows that TiNT catalyst was successfully immobilized on pumice surface. The $\mathrm{Ca}$ and $\mathrm{Fe}$ element were not observed again on the composite, because the amount of both elements were relatively small, thus they could not be detected by the EDX analyze tool.

Table 3 shows the surface area of pure pumice, immobilized TiNT, and TiNT based on the BET results. The surface area of pure pumice is really low compared to the one of TiNT, though pumice has a porous structure. This signifies that the pores on pumice are relatively big compared to the 
nanocatalyst, as can be seen in Fig. 6a, b. Due to the presence of TiNT immobilized on the pumice, the surface area of the products of both methods are larger than pure pumice.

Interestingly, immobilized TiNT-SE has surface area relatively larger than immobilized TiNT-DC, indicating that the product of solvent evaporation method contained relatively more TiNT than the one of dip coating method. This verifies the advantage of solvent evaporation method, in which it could immobilize almost all the particles of catalyst on the support. This also affirms the deduction that solvent evaporation method could deliver the catalyst deeper into the inner segment of the pumice, which was hardly observed by FE-SEM/EDX analysis (as explained before), but on the contrary could be detected by BET analysis. For the case of immobilized $\mathrm{TiO}_{2} \mathrm{P} 25$, it is expected that the result of EDX and BET analysis would be similar to the result of immobilized TiNT, where the main factor determining the result is the immobilization method.

\section{Mechanical strength test}

Table 4 shows the effect of $\mathrm{pH}$ and dispersion medium on the catalyst detachment level of immobilized $\mathrm{TiO}_{2} \mathrm{P} 25-\mathrm{SE}$. At acidic condition, the catalyst detachment level (11.0\%) was lower than the catalyst detachment at normal condition (17.2\%). This phenomenon could happen as the effect of particle size reduction at acidic condition, due to the protonation of $\mathrm{TiO}_{2}$ particles, which would result in better dispersion of $\mathrm{TiO}_{2}$ catalyst on the surface of pumices. It is also said that clay minerals, such as pumice have permanent negative charge on their surface [40, 41]. Hence, there would be great possibility that the electrostatic bond is formed between the positively charged surfaces of $\mathrm{TiO}_{2}$ and negatively charged pumice surfaces. This result proves that controlling the acidity of photocatalyst sol would bring positive effects on the attachment process of titania unto pumice, as explained above.

The usage of ethanol as dispersion medium (on pH 5-6) was able to increase the mechanical strength of titania layer produced, with $11.3 \%$ of catalyst detachment. It is closely related to the stability of the dispersion medium, which has been discussed earlier, where the stability of ethanol

Table 4 Effect of $\mathrm{pH}$ and dispersion medium on the catalyst detachment level of immobilized $\mathrm{TiO}_{2} \mathrm{P} 25-\mathrm{SE}$

\begin{tabular}{lll}
\hline Dispersion medium & $\mathrm{pH}$ & $\begin{array}{l}\text { Catalyst } \\
\text { detachment } \\
(\%)\end{array}$ \\
\hline Aquadest & $5-6$ & 17.2 \\
Aquadest & $2-3$ & 11.0 \\
Ethanol & $5-6$ & 11.3 \\
Ethanol & $2-3$ & 9.5 \\
\hline
\end{tabular}

sol increased dramatically during the evaporation process, whereas the stability of aquadest sol decreased significantly. This outcome confirms that for certain immobilization method, there is a suitable dispersion medium that should be chosen accordingly. When both enhancements were applied (usage of acid condition and ethanol as dispersion medium), the mechanical strength of immobilized titania got even stronger, with only $9.5 \%$ of catalyst detachment.

Table 5 shows the result of mechanical strength test to investigate the effect of immobilization method on catalyst detachment level. For this investigation, optimal condition for each method was also applied regarding to the characteristic of each method: ethanol for solvent evaporation method and aquadest for dip coating method, and for both methods acidic condition was applied. As can be seen, both dip coating method and solvent evaporation method successfully produced immobilized titania on pumice with good mechanical strength (\%catalyst detachment $<10 \%$ ). This could be ascribed to the sol stability, due to the acid condition and appropriate selection of dispersion medium for each method, based on the stability test, as explained before. Stability of the sol would provide favourable condition for titania attaching to pumice surfaces.

The products of dip coating method had slightly lower catalyst detachment level than the products of the solvent evaporation method. This might be attributed to the homogen layer of immobilized titania, which dip coating method could generate, thus making the immobilized titania better able to withstand the external disturbance. On the other hand, for solvent evaporation method, the immobilization process of titania would be random, which gave the possibility for titania to form aggregates on the pumice surface (see Fig. 6c, d). In this case, those aggregates would not be able to hold up against extreme disturbance, making the outer immobilized photocatalyst to detach rather easily. However, the difference between the mechanical strength of those two products is considerably small.

It can also be observed that immobilized TiNT had lower catalyst detachment level compared to immobilized $\mathrm{TiO}_{2}$ $\mathrm{P} 25$. This phenomenon once again demonstrates how nanotube morphology of titania, due to its greater contact area would enhance the mechanical strength of catalyst layer

Table 5 Effect of immobilization method on catalyst detachment level of immobilized titania

\begin{tabular}{ll}
\hline Catalyst & $\begin{array}{l}\text { Catalyst } \\
\text { detachment } \\
(\%)\end{array}$ \\
\hline Immobilized TiNT-SE & 5.0 \\
Immobilized TiNT-DC & 4.6 \\
Immobilized $\mathrm{TiO}_{2}$ P25-SE & 9.5 \\
Immobilized $\mathrm{TiO}_{2}$ P25-DC & 6.7 \\
\hline
\end{tabular}


immobilized on pumice surface compared to nanoparticle. Hence, for further investigation, i.e., the photodegradation performance test, the catalyst used was only immobilized TiNT, considering the prospect of being used for field application due to its good mechanical strength.

\section{Photodegradation performance test}

Similar to the previous sections, the condition used for producing the immobilized TiNT used on this test had been optimized in accordance to the characteristic of each method. Photocatalytic degradation of phenol using pure pumice, TiNT, and immobilized TiNT produced by two different methods were evaluated and the results are presented in Fig. 7a and Table 6. As mentioned before, the kinetic behavior of photocatalytic reactions obeys the pseudo firstorder model $[28,30]$. Hence $\ln \left(C_{t} / C_{0}\right)$ was plotted versus irradiation time (see Fig. $7 \mathrm{~b}$ ) and the rate constants, $k\left(\mathrm{~min}^{-1}\right)$ were also calculated, whose results can also be found on Table 6 .

As can be seen, there are about $5 \%$ of phenol elimination, observed in the presence of pure pumice, indicating an insignificant adsorption of phenol into pumice, due to its porous characteristic. Because of the abundance of Si elements in pumice, its surface tended to be negatively charged [41]. Tapan et al. [40] have reported that pumice has negative $\zeta$-potential for both acid and base conditions ( $\mathrm{pH} 2-12)$ where the isoelectrical point $\left(\mathrm{pH}_{\mathrm{pzc}}\right)$ cannot be observed on any conditions, which confirms that the surface of pumice

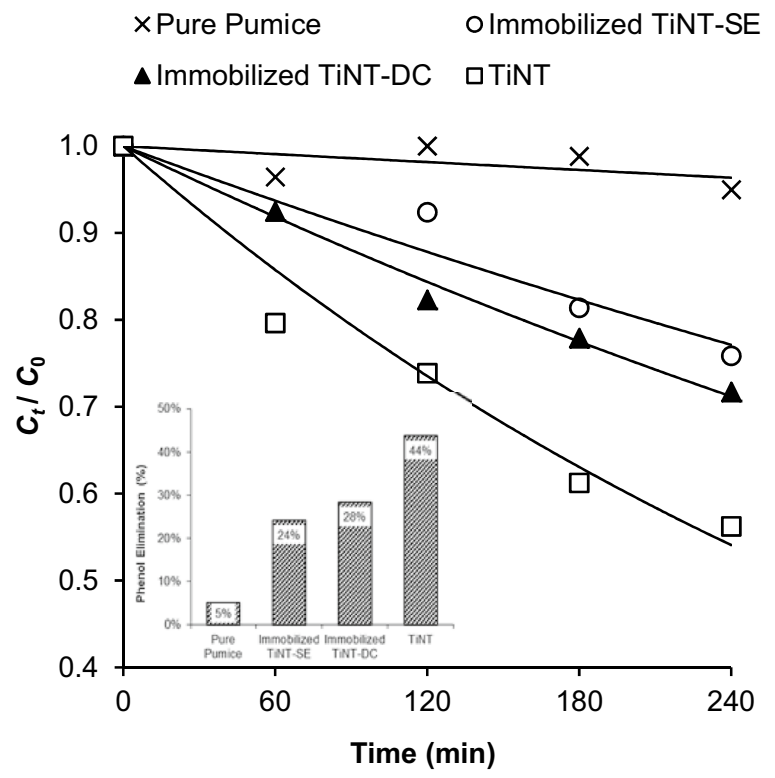

(a)
Table 6 Phenol elimination (at minute-240) and the rate constants $(k)$ of pure pumice, immobilized TiNT, and TiNT

\begin{tabular}{lcl}
\hline Samples & $\begin{array}{l}\text { Phenol elimination } \\
(\%)\end{array}$ & $\begin{array}{l}k \times 1000 \\
\left(\mathrm{~min}^{-1}\right)\end{array}$ \\
\hline Pure pumice & 5 & 0.2 \\
Immobilized TiNT-SE & 24 & 1.1 \\
Immobilized TiNT-DC & 28 & 1.4 \\
TiNT & 46 & 2.6 \\
\hline
\end{tabular}

contains permanent negative charge. $\mathrm{pH}_{\mathrm{pzc}}$ is considered as a point where there will be a change in the surface charge [30]. The negative charge of pumice would cause phenol which also had relatively negative charge due to its hydroxyl group $(-\mathrm{OH})$ to be poorly adsorbed by pumice because of similar charge repulsion. Thus, pumice was only able to adsorb a small portion of phenol pollutant.

Both immobilized TiNT-SE and immobilized TiNT-DC showed similar photocatalytic activity to eliminate phenol, with phenol elimination of 24 and $28 \%$ and rate constant of $1.1 \times 10^{-3} \mathrm{~min}^{-1}$ and $1.4 \times 10^{-3} \mathrm{~min}^{-1}$, respectively. This result proves that both products from two different methods tended to consist same amount of titania, as discussed before. However, the slight difference between the catalyst performances might be ascribed to the different immobilization target area of each method. Dip coating method successfully immobilized the TiNT on the outer pumice surface evenly, which will facilitate the photocatalyst

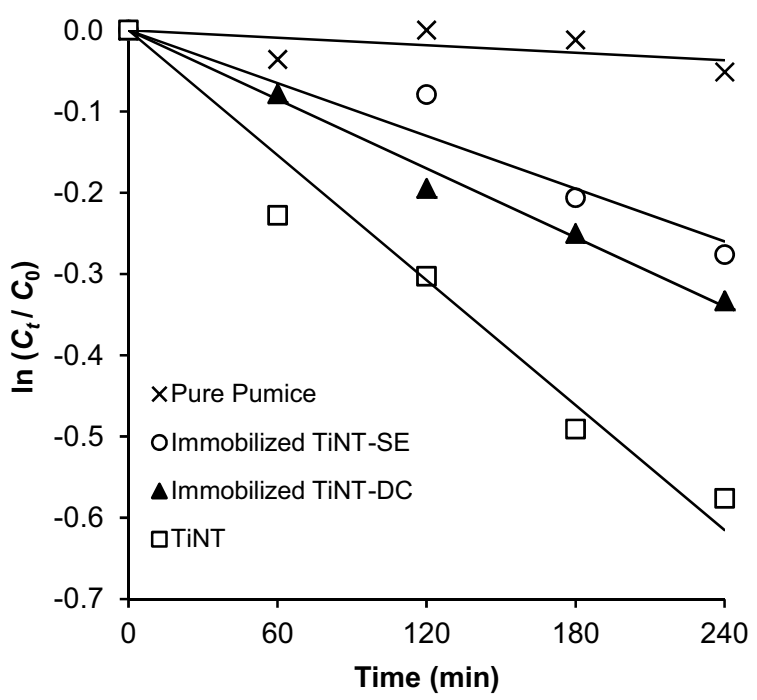

(b)

Fig. 7 a Phenol photodegradation and the degree of phenol elimination (\%) at minute-240, as well as $\mathbf{b}$ plots of $\ln \left(C_{t} / C_{0}\right)$ vs $t$ of pure pumice (multiple sign), immobilized TiNT-SE (open circle), immobilized TiNT-DC (filled triangle), and TiNT (open square) 
to interact with the light more effectively. On the contrary, immobilized TiNT, produced by solvent evaporation method which mostly lodged in the inner pores of pumice would mildly experience shading effect [42] and thus inhibiting the illumination of light which was supposed to activate the photocatalyst. Nevertheless, it was suspected that this result would vary depending on the size of pumice, used as support. Solvent evaporation method is expected to give better result if pumice with smaller size is used, where shading effect can be neglected.

Compared to photocatalyst TiNT itself, performance of immobilized TiNT was relatively lower. This might happen because of fewer contact between TiNT catalyst and phenol pollutant for the immobilized catalyst case, compared to catalyst in slurry condition. On the performance test, immobilized TiNT were only concentrated on the top part of test solution, due to floating ability of pumice. On the other hand, for the performance test of TiNT, the condition was slurry, where contact frequency between catalyst and pollutant was relatively high.

On this point, it is important to understand the purpose for immobilizing the catalyst to floating support, such as pumice, was not to increase the photocatalytic activity of catalyst, but to overcome the regeneration problem of nanosized catalyst. Therefore, especially for field application, the value of photocatalyst is not only determined by its performance to eliminate pollutant but also its ability to be regenerated and used for repeated time. As explained before on the mechanical strength test section, immobilized TiNT had been proven to have good mechanical quality. Hence, it has rather great potential for the field application. Another point to be noted is that the photodegradation performance of immobilized titania might be enhanced by giving certain treatments, like aeration, $\mathrm{pH}$ adjustment, and controlling catalyst loading [1, 43]. For example, an aeration treatment will provide a better mixing condition for the photocatalytic system, hence more contact between immobilized catalyst and the pollutants will likely occur [43]. In addition, it might supply sufficient amount of dissolved oxygen needed for the photodegradation reaction, thus increasing the photocatalytic activity of immobilized titania [43].

For further discussion, the kinetics of phenol photodegradation of the catalysts can be evaluated and used to estimate the time needed for decreasing the pollutant concentration to certain degree. The pseudo first-order kinetic equations of the catalysts are: $\ln \left(C_{t} / C_{0}\right)=-0.0011 t$ for immobilzed TiNT-SEln $\left(C_{t} / C_{0}\right)=-0.0014 t$ for immobilzed TiNTDCln $\left(C_{t} / C_{0}\right)=-0.0026 t$ for TiNT.

For example, based on the kinetic estimation, the time needed for immobilized TiNT-SE, immobilized TiNT-DC, and TiNT to eliminate the phenol to concentration below $0.5 \mathrm{ppm}$ are 45,36 , and $19 \mathrm{~h}$, respectively. The estimated time for pumice is not calculated since its kinetics was really slow.

\section{Conclusion}

Two simple methods (solvent evaporation and dip coating) for immobilizing titania into pumice had been studied and optimized. Based on sol stability and mechanical strength tests, it can be concluded that acid condition would be favourable for immobilizing titania unto pumice. In addition, it was found that for certain immobilization method there is a suitable dispersion medium that should be chosen accordingly: ethanol for solvent evaporation method, while aquadest for dip coating method. Both methods successfully produced not only sol with high level of stability, but also mechanically strong immobilized titania on pumice.

It was also observed that dip coating method tends to produce uniform layer of titania on the outer surface of pumice, whereas solvent evaporation method was apparently capable to deliver titania deeper into internal part of pumice. In this case, shading effect might be encountered for the product of solvent evaporation method, making its photodegradation performance slightly lower than the one of dip coating method. The outcomes of this result suggest that both dip coating and solvent evaporation method could be optimized to produce a promising technology for the elimination of organic pollutants, such as phenol. However, it still needs further improvement and study to find the optimal conditions for the photodegradation system to bring out products which can eliminate waste effectively and efficiently.

Acknowledgements The authors would like to thank DRPM Universitas Indonesia and Directorate General of Higher Education (DGHE) Indonesian Ministry of National Education for the financial support of this research (Hibah PUPT Riset Prioritas, Grant no. 3390/H2.R12/ HKP.05.00/2014).

\section{Compliance with ethical standards}

Conflict of interest The authors declare that they have no conflict of interest.

Open Access This article is distributed under the terms of the Creative Commons Attribution 4.0 International License (http://creativeco mmons.org/licenses/by/4.0/), which permits unrestricted use, distribution, and reproduction in any medium, provided you give appropriate credit to the original author(s) and the source, provide a link to the Creative Commons license, and indicate if changes were made. 


\section{References}

1. Wong CL, Tan YN, Mohamed AR (2011) Photocatalytic degradation of phenol using immobilized nanotube photocatalysts. J Nanotechnol 2011:1-9. https://doi.org/10.1155/2011/904629

2. Nezamzadeh-Ejhieh A, Bahrami M (2015) Investigation of the photocatalytic activity of supported $\mathrm{ZnO}-\mathrm{TiO}_{2}$ on clinoptilolite nano-particles towards photodegradation of wastewater-contained phenol. Desalin Water Treat 55(4):1096-1104. https://doi. org/10.1080/19443994.2014.922443

3. Gondal MA, Seddigi Z (2006) Laser-induced photo-catalytic removal of phenol using n-type $\mathrm{WO}_{3}$ semiconductor catalyst. Chem Phys Lett 417(1-3):124-127. https://doi.org/10.1016/j. cplett.2005.09.115

4. Nezamzadeh-Ejhieh A, Shirzadi A (2014) Enhancement of the photocatalytic activity of Ferrous Oxide by doping onto the nanoclinoptilolite particles towards photodegradation of tetracycline. Chemosphere 107:136-144. https://doi.org/10.1016/j.chemospher e.2014.02.015

5. Bahar K (2016) Effects of Ag, Nd codoping on structural, optical and photocatalytic properties of $\mathrm{TiO}_{2}$ nanocomposite synthesized via sol-gel method using starch as a green additive. Iran J Catal 6(3):305-311

6. Mirian Z-A, Nezamzadeh-Ejhieh A (2016) Removal of phenol content of an industrial wastewater via a heterogeneous photodegradation process using supported $\mathrm{FeO}$ onto nanoparticles of Iranian clinoptilolite. Desalin Water Treat 57(35):16483-16494. https://doi.org/10.1080/19443994.2015.1087881

7. Mousavi-Mortazavi S, Nezamzadeh-Ejhieh A (2016) Supported iron oxide onto an Iranian clinoptilolite as a heterogeneous catalyst for photodegradation of furfural in a wastewater sample. Desalin Water Treat 57(23):10802-10814. https://doi.org/10.1080/19443 994.2015.1036465

8. Fallah Moafi H (2016) Photocatalytic self-cleaning properties of lanthanum and silver co-doped $\mathrm{TiO}_{2}$ nanocomposite on polymeric fibers. Iran J Catal 6(3):281-292

9. Zabihi-Mobarakeh H, Nezamzadeh-Ejhieh A (2015) Application of supported $\mathrm{TiO}_{2}$ onto Iranian clinoptilolite nanoparticles in the photodegradation of mixture of aniline and 2,4-dinitroaniline aqueous solution. J Ind Eng Chem 26:315-321. https://doi. org/10.1016/j.jiec.2014.12.003

10. Akly C, Chadik PA, Mazyck DW (2010) Photocatalysis of gasphase toluene using silica-titania composites: performance of a novel catalyst immobilization technique suitable for large-scale applications. Appl Catal B Environ 99(1-2):329-335. https://doi. org/10.1016/j.apcatb.2010.07.002

11. Slamet Bismo S, Fasa AF, Jabbar A, Putera A (2010) Performance test of a novel tubular V-collector for phenol removal from aqueous solutions over $\mathrm{TiO}_{2}$-activated carbon composites. World Appl Sci J 8(6):672-679

12. Mozia S, Brożek P, Przepiórski J, Tryba B, Morawski AW (2012) Immobilized $\mathrm{TiO}_{2}$ for phenol degradation in a pilot-scale photocatalytic reactor. J Nanomater. https://doi.org/10.1155/2012/94976 4

13. Sopyan I, Hafizah N, Jamal P (2011) Immobilization of $\mathrm{TiO}_{2}$ with cement: photocatalytic degradation of phenol and its kinetic studies. Indian J Chem Technol 18:263-270

14. Lei P, Wang F, Gao X, Ding Y, Zhang S, Zhao J, Liu S, Yang M (2012) Immobilization of $\mathrm{TiO}_{2}$ nanoparticles in polymeric substrates by chemical bonding for multi-cycle photodegradation of organic pollutants. J Hazard Mater 227-228:185-194. https://doi. org/10.1016/j.jhazmat.2012.05.029

15. Bukauskas V, Kaciulis S, Mezzi A, Mironas A, Niaura G, Rudzikas M, Šimkienė I, Šetkus A (2015) Effect of substrate temperature on the arrangement of ultra-thin $\mathrm{TiO}_{2}$ films grown by a dc-magnetron sputtering deposition. Thin Solid Films 585:5-12. https://doi.org/10.1016/j.tsf.2015.04.007

16. Kim D-J, Pham H-C, Park D-W, Kim K-S (2011) Preparation of $\mathrm{TiO}_{2}$ thin films on polypropylene beads by a rotating PCVD process and its application to organic pollutant removal. Chem Eng J 167(1):308-313. https://doi.org/10.1016/j.cej.2010.12.069

17. Balasubramanian G, Dionysiou DD, Suidan MT, Baudin I, Laîné J-M (2004) Evaluating the activities of immobilized $\mathrm{TiO}_{2}$ powder films for the photocatalytic degradation of organic contaminants in water. Appl Catal B Environ 47(2):73-84. https://doi. org/10.1016/j.apcatb.2003.04.002

18. Rachel A, Lavedrine B, Subrahmanyam M, Boule P (2002) Use of porous lavas as supports of photocatalysts. Catal Commun 3(4):165-171. https://doi.org/10.1016/S1566-7367(02)00076-6

19. Magalhães F, Moura FCC, Lago RM (2011) $\mathrm{TiO}_{2} /$ LDPE composites: a new floating photocatalyst for solar degradation of organic contaminants. Desalination 276(1-3):266-271. https:// doi.org/10.1016/j.desal.2011.03.061

20. Zhou M, Roualdès S, Zhao J, Autès V, Ayral A (2015) Nanocrystalline $\mathrm{TiO}_{2}$ thin film prepared by low-temperature plasmaenhanced chemical vapor deposition for photocatalytic applications. Thin Solid Films 589:770-777. https://doi.org/10.1016/j. tsf.2015.07.007

21. Chen L-C, Ke C-R, Hon M-H, Ting J-M (2015) Electrophoretic deposition of $\mathrm{TiO}_{2}$ coatings for use in all-plastic flexible dyesensitized solar cells. Surf Coat Technol 284:51-56. https://doi. org/10.1016/j.surfcoat.2015.07.044

22. Peng Y-P, Yassitepe E, Yeh Y-T, Ruzybayev I, Shah SI, Huang CP (2012) Photoelectrochemical degradation of azo dye over pulsed laser deposited nitrogen-doped $\mathrm{TiO}_{2}$ thin film. Appl Catal B Environ 125:465-472. https://doi.org/10.1016/j.apcatb.2012.06.004

23. Nikazar M, Farahani SJ, Soltani MR (2012) Photocatalytic degradation of ethylene dichloride in water using nano $\mathrm{TiO}_{2}$ supported on clinoptilolite as a photocatalyst. Adv Mater Phys Chem 2:274-276

24. Thiruvenkatachari R, Vigneswaran S, Moon I (2008) A review on $\mathrm{UV} / \mathrm{TiO}_{2}$ photocatalytic oxidation process (Journal Review). Korean J Chem Eng 25(1):64-72. https://doi.org/10.1007/s1181 4-008-0011-8

25. Su C, Hong BY, Tseng CM (2004) Sol-gel preparation and photocatalysis of titanium dioxide. Catal Today 96(3):119-126. https ://doi.org/10.1016/j.cattod.2004.06.132

26. Slamet Nasution HW, Purnama E, Kosela S, Gunlazuardi J (2005) Photocatalytic reduction of $\mathrm{CO}_{2}$ on copper-doped titania catalysts prepared by improved-impregnation method. Catal Commun 6(5):313-319. https://doi.org/10.1016/j.catcom.2005.01.011

27. Slamet Tristantini D, Valentina Ibadurrohman M (2013) Photocatalytic hydrogen production from glycerol-water mixture over Pt-N-TiO 2 nanotube photocatalyst. Int J Energy Res 37(11):1372-1381. https://doi.org/10.1002/er.2939

28. Azimi S, Nezamzadeh-Ejhieh A (2015) Enhanced activity of clinoptilolite-supported hybridized PbS-CdS semiconductors for the photocatalytic degradation of a mixture of tetracycline and cephalexin aqueous solution. J Mol Catal A Chem 408:152-160. https://doi.org/10.1016/j.molcata.2015.07.017

29. Khodami Z, Nezamzadeh-Ejhieh A (2015) Investigation of photocatalytic effect of $\mathrm{ZnO}-\mathrm{SnO}_{2} /$ nano clinoptilolite system in the photodegradation of aqueous mixture of 4-methylbenzoic acid/2chloro-5-nitrobenzoic acid. J Mol Catal A Chem 409:59-68. https ://doi.org/10.1016/j.molcata.2015.08.013

30. Arabpour N, Nezamzadeh-Ejhieh A (2015) Modification of clinoptilolite nano-particles with iron oxide: increased composite catalytic activity for photodegradation of cotrimaxazole in aqueous suspension. Mater Sci Semicond Process 31((Complete)):684-692. https://doi.org/10.1016/j.mssp.2014.12.067 
31. Spurr RA, Myers H (1957) Quantitative analysis of anatase-rutile mixtures with an X-ray diffractometer. Anal Chem 29(5):760-762. https://doi.org/10.1021/ac60125a006

32. Qamar M, Yoon CR, Oh HJ, Lee NH, Park K, Kim DH, Lee KS, Lee WJ, Kim SJ (2008) Preparation and photocatalytic activity of nanotubes obtained from titanium dioxide. Catal Today 131(14):3-14. https://doi.org/10.1016/j.cattod.2007.10.015

33. Sun J, Gao L (2003) Development of a dispersion process for carbon nanotubes in ceramic matrix by heterocoagulation. J Carbon 41:1063-1068

34. Wang B, Xue D, Shi Y, Xue F (2008) Titania 1D nanostructured materials: synthesis, properties and applications. In: Prescott WV, Schwartz AI (eds) Nanorods, Nanotubes and Nanomaterials Research Progress. Nova Science Publishers Inc, New York, pp 163-201

35. Quantachrome Corp (1992) User manual of Autosorb-6B, automated surface area and pore size analyzer. Quantachrome Corp., New York

36. Schaffer $\mathbf{J}$ (2012) Immobilization of $\mathrm{TiO}_{2}$ via different routes for photocatalytic reactions in a PDMS based microreactor. Dissertation, University of Twente, Enschede

37. Li XW, Song RG, Jiang Y, Wang C, Jiang D (2013) Surface modification of $\mathrm{TiO}_{2}$ nanoparticles and its effect on the properties of fluoropolymer/ $\mathrm{TiO}_{2}$ nanocomposite coatings. Appl Surf Sci 276:761-768. https://doi.org/10.1016/j.apsusc.2013.03.167

38. Körlü AE, Yapar S, Perinçek S, Yılmaz H, Bağıran C (2015) Dye removal from textile waste water through the adsorption by pumice used in stone washing. AUTEX Res J 15(3):158-163. https://doi.org/10.1515/aut-2015-0012

39. Tsai C-C, Teng H (2004) Regulation of the physical characteristics of titania nanotube aggregates synthesized from hydrothermal treatment. Chem Mater 16(22):4352-4358

40. Tapan M, Depci T, Özvan A, Efe T, Oyan V (2013) Effect of physical, chemical and electro-kinetic properties of pumice on strength development of pumice blended cements. Mater Struct 46(10):1695-1706. https://doi.org/10.1617/s11527-012-0008-y

41. Akbal F (2005) Sorption of phenol and 4-chlorophenol onto pumice treated with cationic surfactant. J Environ Manag 74:239-244

42. Gulyas H, Argáez ÁSO, Kong F, Jorge CL, Eggers S, Otterpohl R (2013) Combining activated carbon adsorption with heterogeneous photocatalytic oxidation: lack of synergy for biologically treated greywater and tetraethylene glycol dimethyl ether. Environ Technol 34(11):1393-1403. https://doi.org/10.1080/09593 330.2012.751129

43. Lam S-M, Sin J-C, Mohamed A (2010) Parameter effect on photocatalytic degradation of phenol using $\mathrm{TiO}_{2}-\mathrm{P} 25 /$ activated carbon (AC). Korean J Chem Eng 27(4):1109-1116. https://doi. org/10.1007/s11814-010-0169-8

Publisher's Note Springer Nature remains neutral with regard to jurisdictional claims in published maps and institutional affiliations. 